\title{
Multiscale Splitting Method for the Boltzmann-Poisson Equation: Application to the Dynamics of Electrons
}

\author{
Jürgen Geiser \\ Department of Physics, Ernst-Moritz-Arntz University of Greifswald, Domstraße 14, 17487 Greifswald, Germany \\ Correspondence should be addressed to Jürgen Geiser; geiser@mathematik.hu-berlin.de
}

Received 15 August 2013; Revised 20 January 2014; Accepted 21 January 2014; Published 3 March 2014

Academic Editor: Shuyu Sun

Copyright (c) 2014 Jürgen Geiser. This is an open access article distributed under the Creative Commons Attribution License, which permits unrestricted use, distribution, and reproduction in any medium, provided the original work is properly cited.

\begin{abstract}
We present a model based on dynamics of electrons in a plasma using a simplified Boltzmann equation coupled with Poisson's equation. The motivation arose from simulating active plasma resonance spectroscopy, which is used for plasma diagnostic techniques; see Braithwaite and Franklin (2009), Lapke et al. (2010), and Oberrath et al. (2011). Mathematically, we are interested in designing splitting methods for the model problem. While the full Boltzmann equation is delicate to solve, we decouple it into a transport and collision part, which are then solved in different ways. First we reduce it to a simplified transport-collision equation and start to analyse the abstract Cauchy problem using semigroup methods. Second, we pass to the coupled transport and collision model and apply the splitting ideas, resecting the different discretization schemes. The results are discussed first with numerical experiments and then we verify the underlying theoretical novelties.
\end{abstract}

\section{Introduction}

Our motive arose from studying the simulation of active plasma resonance spectroscopy, a well-established plasma diagnostic technique. To study this technique with simulation models, we concentrate on an abstract kinetic model that describes the dynamics of the electrons in plasma by using a Boltzmann equation. The Boltzmann equation is coupled with the electric field and we obtain coupled partial differential equations. The full Boltzmann equation is a very delicate equation to solve. Therefore, we decouple this into transport and collision contributions, which are solved in different ways. While finite difference schemes are applied to the transport parts, the collision part is solved with numerical integration schemes. The underlying splitting scheme is theoretically discussed as an abstract Cauchy problem. Here, we could address the Cauchy problem with functional analytical tools. First, we will discuss the description of a positive semigroup, which helps carry out the numerical estimates in the splitting schemes. Second, a numerical method is discussed with respect to separate differential and integral parts of the equations. The numerical approximation of the abstract splitting scheme is made by applying an iterative splitting method of the second order.
The paper is outlined as follows. In Section 2, we present our mathematical model and a possible reduced model for further approximations. The functional analytical setting with semigroups is discussed in Section 3. The splitting schemes are presented in Section 4. The results of some numerical experiments are exhibited in Section 6. In the conclusions that are given in Section 7, we summarize our results.

\section{Mathematical Model}

In the following a model is presented whose physical motivation is explained in [1-3].

The kinetic model considers a fluid dynamical approach to treat the natural ability of plasma to resonate near the electron plasma frequency $\omega_{\text {pe }}$.

Here we specialise in an abstract kinetic model to describe the dynamics of the electrons in the plasma which allows for this resonance.

The Boltzmann equation for the electrons is

$$
\begin{array}{r}
\frac{\partial f(x, v, t)}{\partial t}=\mathscr{A}[f(x, v, t)]+\mathscr{B}[f(x, v, t)], \\
(x, v) \in \Omega_{x} \times \Omega_{v}, \quad t \in[0, T],
\end{array}
$$




$$
\begin{gathered}
\mathscr{A}[f]=-v \cdot \nabla_{x} f(x, v, t)-\frac{e}{m_{e}} \nabla_{x} \phi \cdot \nabla_{v} f(x, v, t), \\
\mathscr{B}[f]=-\sigma(x, v, t) f(x, v, t)+\int_{V} \kappa\left(x, v, v^{\prime}\right) f\left(x, v^{\prime}, t\right) d v^{\prime}, \\
f(x, v, 0)=f_{0}(x, v), \quad(x, v) \in \Omega_{P, x} \times \Omega_{P, v}, \\
f(x, v, t)=f_{1}(x, v), \quad(x, v) \in \partial \Omega_{P, x} \times \partial \Omega_{P, v},
\end{gathered}
$$

where $\Omega_{P, x} \times \Omega_{P, v} \subset \mathbb{R}^{3} \times \mathbb{R}^{3}$ is the six-dimensional phase space. $f: \Omega_{P, x} \times \Omega_{P, v} \times[0, T] \rightarrow \mathbf{X}_{c}$ is the density function and $\mathbf{X}_{c}$ is an appropriate smooth space; for example, $C_{2} . f_{0}$ is the initial function and the $f_{1}$ is the boundary condition in the plasma phase space $\Omega_{P, x} \times \Omega_{P, y}$.

We assume based on the different materials (a plasma and a dielectricum) a complete reflection of the electrons due to the sheath $f\left(v_{\|}+v_{\perp}\right)$ with $v_{\|}$, which is the parallel, and $v_{\perp}$ which is perpendicular to the surface normal vector. Further the electric force field is given as $F=e \nabla_{x} \phi$, where $\phi: \Omega_{P, x} \rightarrow$ $\mathbf{X}_{c}$ is the potential and $\mathbf{X}_{c}$ is an appropriate smooth space, for example, $C_{2}$.

Boltzmann's equation is coupled with the electric field $\nabla \phi$, while the potential is approximated via Poisson's equation. The electrostatic approximation of the field is represented by a potential that is valid on the complete velocity volume $S \subset \Omega_{P, v}$, and apply Poisson's equation:

$$
-\nabla_{x} \cdot(\epsilon \nabla \phi)= \begin{cases}e\left(n_{i}(x)-\int f d S\right) & \text { in } x \in \Omega_{P, x} \\ 0 & \text { in } x \in \Omega_{D, x},\end{cases}
$$

where the full space domain is $\Omega_{x}=\Omega_{P, x} \cup \Omega_{D, x} \subset \mathbb{R}^{3}$ and the full velocity domain is $\Omega_{v}=\Omega_{P, v} \cup \Omega_{D, v}$. The permittivity is equal to $\epsilon_{0}$ in the plasma $\Omega_{P, x}$ and $\epsilon_{0} \epsilon_{D}$ in the dielectric $\Omega_{D, x}$. $\phi$ fulfills the boundary conditions $U_{n}$ at any electrode $E_{n}$ and $-\mathbf{n} \cdot \nabla \phi=0$ at isolator $I$, whereas $\mathbf{n}$ is the normal vector of the isolator surface.

On the surface of the dielectric, a surface charge $\sigma$ may accumulate, which leads to a transition condition

$$
\Delta(\epsilon \nabla \phi)=-\sigma .
$$

We will make the following assumption to modify the model into a simpler and more tractable system of equations, which allows linearizing and simplifying the Boltzmann equations; see [4].

First we assume that we have an analytical solution of the potential $\phi$, such that we could replace the convective term of the velocity-variable to a diffusive term of the space-variable as

$$
\frac{F}{m_{e}} \cdot \nabla_{v} \approx \nabla_{x} D \nabla_{x}
$$

where $D$ is the diffusion tensor and $F=e \nabla \phi$ is the external force. This modified model allows employing the following semigroup theory and deriving splitting schemes for the model.
The modified and splittable model equation is

$$
\begin{gathered}
\frac{\partial f(x, v, t)}{\partial t}=\mathscr{A}[f(x, v, t)]+\mathscr{B}[f(x, v, t)], \\
(x, v) \in \Omega_{x} \times \Omega_{v}, \quad t \in[0, T], \\
\mathscr{A}[f]=-v \cdot \nabla_{x} f(x, v, t)-\nabla_{x} D \nabla_{x} f(x, v, t), \\
\mathscr{B}[f]=-\sigma(x, v, t) f(x, v, t)+\int_{V} \kappa\left(x, v, v^{\prime}\right) f\left(x, v^{\prime}, t\right) d v^{\prime}, \\
f(x, v, 0)=f_{0}(x, v), \quad(x, v) \in \Omega_{P, x} \times \Omega_{P, v}, \\
f(x, v, t)=f_{1}(x, v), \quad(x, v) \in \partial \Omega_{P, x} \times \partial \Omega_{P, v} .
\end{gathered}
$$

Next, we will discuss the relevant semigroup theory.

\section{Semigroups for Transport Equations}

In the following, we derive the exponential growth of the transport semigroups that are used in the section on the numerical methods.

We discuss two aspects:

(i) neutron transport,

(ii) electron transport.

The differences of the two transport schemes are as follows.

(i) Neutron transport is not influenced by an electric field.

(ii) Electron transport is influenced by an electric field.

3.1. Transport Model for the Neutrons. For this model we can assume that $f(x, v, t)$ describes the density distribution of the particles at position $x \in S$ with speed $v \in V$ at time $t \in[0, T]$; see also $[5,6]$.

The space $S$ is assumed to be a compact and convex subset of $\mathbb{R}^{3}$ with nonempty interior, and the velocity space $V$ is

$$
V:=\left\{v \in \mathbb{R}^{3}: v_{\min } \leq\|\|_{2} \leq v_{\max }\right\},
$$

for $v_{\min }>0$ and $v_{\max }<\infty$.

Assumption 1. We make the following assumptions.

(i) Particles move according to their speed $v$.

(ii) Particles are absorbed according to the probability given by the function $\sigma$ which depends on $x$ and $v$.

(iii) Particles are scattered according to a scattering kernel $\kappa$ depending on $x$, the incoming speed $v^{\prime}$, and the outgoing speed $v$.

Then the neutron transport is

$$
\begin{aligned}
\frac{\partial f(x, v, t)}{\partial t}= & -v \cdot \nabla f(x, v, t)-\sigma(x, v, t) f(x, v, t) \\
& +\int_{V} \kappa\left(x, v, v^{\prime}\right) f\left(x, v^{\prime}, t\right) d v^{\prime}, \\
& f(x, v, 0)=f_{0}(x, v),
\end{aligned}
$$


and boundary conditions are included in the transport operator $A_{0}$; see, in the following, the abstract Cauchy problem.

We next treat the abstract Cauchy problem for this simplified model.

Abstract Cauchy Problem: Transport Model for the Neutrons. We have a Banach space $\mathbf{X}:=L^{1}(S \times V)$ with Lebesgue measure on $S \times V \subset \mathbb{R}^{6}$ and define the abstract Cauchy problem as

$$
\begin{gathered}
\frac{d u(t)}{d t}=B u(t), \\
\frac{d u(t)}{d t}=\left(A_{0}-M_{\sigma}+K_{\kappa}\right) u(t), \\
u(0)=u_{0},
\end{gathered}
$$

where $u \in \mathbf{X}$

We have the following operators:

(1) collisionless transport operator,

(2) absorption operator,

(3) scattering Operator.

Important results for the further numerical analysis is the fact that the transport semigroup can be estimated by an exponential growth; see [5].

Corollary 2. One assumes that $s(B)>-\infty$ is a dominant eigenvalue and $(S(t))_{t \geq 0}$ is irreducible. Then the transport semigroup $(S(t))_{t \geq 0}$ has balanced exponential growth. There exists a one-dimensional projection $P$ satisfying $0<P f$ whenever $0<f$ such that

$$
\|\exp (-s(B) t) S(t)-P\| \leq M \exp (-\epsilon t),
$$

for all $t \geq 0$ and appropriate $M \geq 1$ and $\epsilon>0$.

3.2. Transport Model for the Electrons or Ions. For this model we can assume that $f(x, v, t)$ describes the density distribution of particles at position $x \in S$ with speed $v \in V$ at time $t \in[0, T]$; see also $[5,6]$.

The space $S$ is assumed to be a compact and convex subset of $\mathbb{R}^{3}$ with nonempty interior, and the velocity space $V$ is

$$
V:=\left\{v \in \mathbb{R}^{3}: v_{\min } \leq\|\|_{2} \leq v_{\max }\right\},
$$

for $v_{\min }>0$ and $v_{\max }<\infty$.

Assumption 3. We make the following assumptions.

(i) Particles move according to their speed $v$.

(ii) Particles are absorbed according to the probability given by the function $\sigma$ which depends on $x$ and $v$.

(iii) Particles are scattered according to a scattering kernel $\kappa$ depending on $x$, the incoming speed $v^{\prime}$, and the outgoing speed $v$.

(iv) Particles are influenced by the static electric field $\phi$, which can be derived by the kinetic theory.
The electron transport is

$$
\begin{aligned}
& \frac{\partial f(x, v, t)}{\partial t} \\
& =-v \cdot \nabla_{x} f(x, v, t)-\frac{e}{m_{e}} \nabla_{x} \phi \cdot \nabla_{v} f(x, v, t) \\
& -\sigma(x, v, t) f(x, v, t)+\int_{V} \kappa\left(x, v, v^{\prime}\right) f\left(x, v^{\prime}, t\right) d v^{\prime}, \\
& f(x, v, 0)=f_{0}(x, v),
\end{aligned}
$$

and boundary conditions are included in the transport operators. $\phi$ is the electric field.

Furthermore, we have Poisson's equation,

$$
-\nabla_{x} \cdot(\epsilon \nabla \phi)= \begin{cases}e\left(n_{i}-\int f d S\right) & \text { in } P \\ 0 & \text { in } D\end{cases}
$$

and the permittivity is equal to $\epsilon_{0}$ in the plasma $P$ and $\epsilon_{0} \epsilon_{D}$ in the dielectric $D$.

For the simplification, we assume to solve Poisson's equation analytically with

$$
\begin{aligned}
\phi & =\int_{\Omega} \mathscr{D}(f) d x \\
& = \begin{cases}\int_{\Omega_{P}} \epsilon^{-1} \int_{\Omega_{P}} e\left(n_{i}-\int f d S\right) d x d x & \text { in } P \\
0 & \text { in } D,\end{cases}
\end{aligned}
$$

where $\mathscr{D}$ is the analytical function of the electric field $\nabla_{x} \phi$.

We embed the electric field analytically into the transport equation

$$
\begin{aligned}
& \frac{\partial f(x, v, t)}{\partial t} \\
& =-v \cdot \nabla_{x} f(x, v, t)-\nabla_{x} \mathscr{D}(f) \cdot \nabla_{x} f(x, v, t) \\
& -\sigma(x, v, t) f(x, v, t)+\int_{V} \kappa\left(x, v, v^{\prime}\right) f\left(x, v^{\prime}, t\right) d v^{\prime}, \\
& f(x, v, 0)=f_{0}(x, v),
\end{aligned}
$$

and boundary conditions are included in the transport operators $A_{0}$ and $A_{1}$; see, in the following, the treatment of the abstract Cauchy problem. $\mathscr{D}(f)$ is the diffusion parameter that includes the electric field and we assume to approximate it via a constant operator $\mathscr{D} \approx \mathscr{D}(f)$.

Next we treat the abstract Cauchy problem for a transport model for the electrons or ions.

Abstract Cauchy Problem: Transport Model for the Electrons or Ions. We have a Banach space $\mathbf{X}:=L^{1}(S \times V)$ with Lebesgue 
measure on $S \times V \subset \mathbb{R}^{6}$ and define the abstract Cauchy problem as

$$
\begin{gathered}
\frac{d u(t)}{d t}=B u(t), \\
\frac{d u(t)}{d t}=\left(A_{0}+A_{1}-M_{\sigma}+K_{\kappa}\right) u(t), \\
u(0)=u_{0},
\end{gathered}
$$

where $u \in \mathbf{X}$.

We have the following operators:

(1) collisionless transport operator,

(2) diffusion operator,

(3) absorption operator,

(4) scattering Operator.

An important result for the further numerical analysis is the fact that the transport semigroup can be estimated by an exponential growth. Due to the analytical embedding of the electric field, we could also estimate such operator.

Corollary 4. One assumes that $s(\widetilde{B})>-\infty$ is a dominant eigenvalue and $(S(t))_{t \geq 0}$ is irreducible. Then the transport semigroup $(S(t))_{t \geq 0}$ has balanced exponential growth. There exists a one-dimensional projection $\widetilde{P}$ satisfying $0<\widetilde{P} f$ whenever $0<f$ such that

$$
\|\exp (-s(\widetilde{B}) t) S(t)-\widetilde{P}\| \leq \widetilde{M} \exp (-\epsilon t),
$$

for all $t \geq 0$ and appropriate $\widetilde{M} \geq 1$ and $\epsilon>0$.

In the next section we discuss the splitting schemes.

\section{Splitting Schemes}

In general, operator splitting methods are used to solve complex models in geophysical and environmental physics. They have been developed and applied in [7-9].

4.1. Sequential Splitting Method for Nonlinear Problems. For our problems, nonlinear splitting schemes are necessary; see [10]. We could use the result for the general formulation of nonlinear ordinary differential equations, which are given by

$$
c^{\prime}(t)=F_{1}(t, c(t))+F_{2}(t, c(t)),
$$

where the initial conditions are $c^{n}=c\left(t^{n}\right)$.

As before, we can decouple the above problem into two (usually simpler) subproblems, namely,

$$
\begin{gathered}
\frac{\partial c^{*}(t)}{\partial t}=F_{1}\left(t, c^{*}(t)\right) \\
\text { with } t^{n} \leq t \leq t^{n+1}, \quad c^{*}\left(t^{n}\right)=c^{n}, \\
\frac{\partial c^{* *}(t)}{\partial t}=F_{2}\left(t, c^{* *}(t)\right)
\end{gathered}
$$

with $t^{n} \leq t \leq t^{n+1}, \quad c^{* *}\left(t^{n}\right)=c^{*}\left(t^{n+1}\right)$, where the initial values are given by $c^{n}=c\left(t^{n}\right)$ and the split approximation on the next time level is defined as $c^{n+1}=$ $c^{* *}\left(t^{n+1}\right)$.

For this case, the splitting error can be determined by using the Jacobians of the nonlinear mappings $F_{1}$ and $F_{2}$ :

$$
\rho_{n}=\frac{1}{2} \tau\left[\frac{\partial F_{1}}{\partial c} F_{2}, \frac{\partial F_{2}}{\partial c} F_{1}\right]\left(t^{n}, c\left(t^{n}\right)\right)+\mathcal{O}\left(\tau_{n}^{2}\right) .
$$

Hence, for the general case, the splitting error is of first order, that is, $O\left(\tau_{n}\right)$.

Remark 5. Higher order splitting methods are given in [11]. Based on the Strang splitting, higher order nonlinear splitting methods are also possible; see [12].

In the next subsection we present the iterative splitting method.

4.2. Iterative Splitting Method. Alternatives are iterative approaches to nonlinear splitting schemes.

We concentrate again on nonlinear differential equations of the form

$$
\begin{array}{r}
\frac{d u}{d t}=A(u(t)) u(t)+B(u(t)) u(t), \\
\text { with } u\left(t^{n}\right)=u^{n},
\end{array}
$$

where $A(u), B(u)$ are matrices with nonlinear entries and densely defined, where we assume that the entries involve the spatial derivatives of $c$; see [13]. In the following we discuss the standard iterative operator splitting method as a fixed-point iteration method to linearize the operators.

We split our nonlinear differential equation (20) by applying

$$
\begin{array}{r}
\frac{d u_{i}(t)}{d t}=A\left(u_{i-1}(t)\right) u_{i}(t)+B\left(u_{i-1}(t)\right) u_{i-1}(t), \\
\text { with } u_{i}\left(t^{n}\right)=c^{n}, \\
\frac{d u_{i+1}(t)}{d t}=A\left(u_{i-1}(t)\right) u_{i}(t)+B\left(u_{i-1}(t)\right) u_{i+1}(t), \\
\text { with } u_{i+1}\left(t^{n}\right)=c^{n},
\end{array}
$$

where the time step is $\tau=t^{n+1}-t^{n}$. The iterations are $i=$ $1,3, \ldots, 2 m+1 \cdot u_{0}(t)=c_{n}$ is the starting solution, where we assume that the solution $c^{n+1}$ is near $c^{n}$ or $u_{0}(t)=0$. Thus we have to solve the local fixed-point problem. $c^{n}$ is the known split approximation at time level $t=t^{n}$.

The split approximation at time level $t=t^{n+1}$ is defined as $c^{n+1}=u_{2 m+2}\left(t^{n+1}\right)$. We assume that the operators $A\left(u_{i-1}\left(t^{n+1}\right)\right), B\left(u_{i-1}\left(t^{n+1}\right)\right)$ are constant for $i=1,3, \ldots, 2 m+$ 1. Here the linearization is done with respect to the iterations, such that $A\left(u_{i-1}\right), B\left(u_{i-1}\right)$ are at least nondependent operators in the iterative equations, and we can apply the linear theory. For the linearization we assume that at least in the first equation $A\left(u_{i-1}(t)\right) \approx A\left(u_{i}(t)\right)$ and in the second equation $B\left(u_{i-1}(t)\right) \approx B\left(u_{i+1}(t)\right)$, for small $t$. 
We have

$$
\left\|A\left(u_{i-1}\left(t^{n+1}\right)\right) u_{i}\left(t^{n+1}\right)-A\left(u^{n+1}\right) u\left(t^{n+1}\right)\right\| \leq \epsilon,
$$

for sufficient iterations $i \in\{1,3, \ldots, 2 m+1\}$.

Remark 6. The linearization with the fixed-point scheme can be used for smooth or weakly nonlinear operators, but otherwise we lose the convergence behavior, not converging to a local fixed point; see [14].

\section{Numerical Integration of the Integrodifferential Part}

We treat the following integrodifferential equation:

$$
\begin{gathered}
\frac{\partial u}{\partial t}=\int_{0}^{t} u(s) d s, \\
u(0)=u_{0} .
\end{gathered}
$$
rule:

The integration part is done numerically with trapezoidal

$$
\begin{aligned}
& \int_{a}^{b} f(x) d x \\
& \quad \approx \frac{b-a}{n}\left(\frac{f(a)+f(b)}{2}+\sum_{k=1}^{n-1} f\left(a+k \frac{b-a}{n}\right)\right),
\end{aligned}
$$

where the subintervals have the form $[k h,(k+1) h]$, with $h=$ $(b a) / n$ and $k=0,1,2, \ldots, n 1$.

The higher order formulas are given as closed NewtonCotes formulas, where $f_{i}$ is a shorthand for $f\left(x_{i}\right)$, with $x_{i}=$ $a+i(b-a) / n$, and $n$ is the degree. We obtain the following formula for the trapezoid rule:

$$
\begin{gathered}
\frac{\partial u}{\partial t}=\frac{t}{2(u(0)+u(t))} d s, \\
u(0)=u_{0},
\end{gathered}
$$

and obtain the analytical result

$$
u(t)=\frac{2}{2} \exp \left(\frac{t^{2}}{4}\right) u(0)-\frac{1}{2} u(0) .
$$

For a higher order formula like Simpson's rule, we have

$$
\begin{gathered}
\frac{\partial u}{\partial t}=\frac{t}{6(u(0)+4 u(t / 2)+u(t))} d s, \\
u(0)=u_{0} .
\end{gathered}
$$

We apply the idea of a polynomial solution:

$$
u(t)=a_{0}+a_{1} t+a_{2} t^{2}+a_{3} t^{3}+\cdots,
$$

and we obtain, after differentiating the coefficients,

$$
\begin{gathered}
a_{1}+2 a_{2} t+3 a_{3} t^{2}+\cdots \\
=t\left(6 \left(a_{0}+4\left(a_{0}+\frac{a_{1} t}{2}+\frac{a_{2} t^{2}}{4}+\frac{a_{3} t^{3}}{8}+\cdots\right)\right.\right. \\
\left.\left.+a_{0}+a_{1} t+a_{2} t^{2}+a_{3} t^{3}+\cdots\right)\right)^{-1} \\
a_{0}=u_{0} .
\end{gathered}
$$

Now, comparing coefficients yields

$$
\begin{gathered}
a_{0}=u_{0}, \\
a_{1}=a_{3}=a_{5}=\cdots=0, \\
a_{2}=3 a_{0}, \\
a_{4}=\frac{1}{12} a_{2}, \ldots
\end{gathered}
$$

\section{Numerical Experiments}

We present the results of our numerical experiments based on the neutron transport. A simplified one-dimensional model is given by

$$
\partial_{t} c+v \partial_{x} c-D \partial_{x x} c+\sigma c=\int_{\Omega} \kappa\left(x, v, v^{\prime}\right) c\left(x, v^{\prime}, t\right) d v^{\prime} .
$$

The velocity $v$ and the diffusion $D$ are given by the plasma model. The initial conditions are given by $c(x, 0)=c_{0}(x)$ and the boundary conditions are trivial; $\partial_{n} c(x, t)=0$.

A first integral operator is

$$
\int_{\Omega} \kappa\left(x, v, v^{\prime}\right) c\left(x, v^{\prime}, t\right) d v^{\prime}=\int_{0}^{T} c(x, t) d t .
$$

A second integral operator is as follows.

We assume a simple collision operator $\kappa\left(x, v, v^{\prime}\right)=$ $q\left(v^{\prime}\right)\left(1+v^{\prime 2}\right)$, where $q\left(v^{\prime}\right)$ is the potential, for example, $v^{\prime 2}$.

We deal with the first integral operator and define the following operators:

$$
\begin{gathered}
A=v \frac{1}{\Delta x}\left[\begin{array}{ccc}
-1 & 1 & 0
\end{array}\right] I-D \frac{1}{\Delta x^{2}}\left[\begin{array}{lll}
1 & -2 & 1
\end{array}\right] I, \\
B=(-\sigma+t) I,
\end{gathered}
$$

while

$$
\exp (B t)=\exp \left(\left(-\sigma t+\frac{t^{2}}{2}\right) I\right)
$$

where $I$ is the identity matrix.

In the following, the simplified real-life problem for a neutron transport equation, which includes the gain and loss of a neutron, will be presented. 
We concentrate on the computational benefits of a fast computation of the iterative scheme, given with matrix exponentials.

The equation is

$$
\begin{gathered}
\partial_{t} c+\nabla \cdot \mathbf{F} c=-\lambda_{1} c+\int_{0}^{t} \lambda_{2} c(x, t) d t, \quad \text { in } \Omega \times[0, t], \\
\mathbf{F}=\mathbf{v}-D \nabla, \\
c(\mathbf{x}, t)=c_{0}(\mathbf{x}), \quad \text { on } \Omega, \\
c(\mathbf{x}, t)=c_{1}(\mathbf{x}, t), \quad \text { on } \partial \Omega \times[0, t] .
\end{gathered}
$$

In the following, we deal with the semidiscretized equation given by the matrices

$$
\partial_{t} \mathbf{C}=\left(A-\Lambda_{1}+\Lambda_{2}\right) \mathbf{C},
$$

where $\mathbf{C}=\left(c_{1}, \ldots, c_{I}\right)^{T}$ is the solution of the species in the mobile phase in each spatial discretization point $(i=$ $1, \ldots, I)$.

We have the following two operators for the splitting method:

$$
\begin{aligned}
A & =\frac{D}{\Delta x^{2}} \cdot\left(\begin{array}{ccccc}
-2 & 1 & & & \\
1 & -2 & 1 & & \\
& \ddots & \ddots & \ddots & \\
& & 1 & -2 & 1 \\
& & & 1 & -2
\end{array}\right) \\
& +\frac{v}{\Delta x} \cdot\left(\begin{array}{ccccc}
1 & & & & \\
-1 & 1 & & & \\
& \ddots & \ddots & & \\
& & -1 & 1 & \\
& & & -1 & 1
\end{array}\right) \in \mathbb{R}^{I \times I},
\end{aligned}
$$

$$
\Lambda_{2}\left(\mathbf{C}_{i-1}\right)=\left(\begin{array}{cccc}
\int_{0}^{t} \lambda_{2} c_{1, i-1}(x, s) d s & 0 & \cdots & 0 \\
0 & \int_{0}^{t} \lambda_{2} c_{2, i-1}(x, s) d s & 0 & \\
\vdots & \ddots & \ddots & \vdots \\
0 & \cdots & 0 \int_{0}^{t} \lambda_{2} c_{I, i-1}(x, s) d s
\end{array}\right) \in \mathbb{R}^{I \times I} .
$$

We obtain $B(\mathbf{C})=\Lambda_{2}\left(\mathbf{C}_{i-1}\right)+\Lambda_{1} \mathbf{C}$.

The iterative scheme is given by the following.

For $i=1,2, \ldots$

$$
\begin{aligned}
\mathbf{C}_{i}(t)= & \exp \left(A\left(t-t^{n}\right)\right) \mathbf{C}\left(t^{n}\right) \\
& +\int_{t^{n}}^{t} \exp ((t-s) A) B\left(\mathbf{C}_{i-1}(s)\right) d s, \quad t \in\left(t^{n}, t^{n+1}\right] .
\end{aligned}
$$

where $I$ is the number of spatial points. Consider the following:

$$
\Lambda_{1}=\left(\begin{array}{ccccc}
\lambda_{1} & 0 & & & \\
0 & \lambda_{1} & 0 & & \\
& \ddots & \ddots & \ddots & \\
& & 0 & \lambda_{1} & 0 \\
& & & 0 & \lambda_{1}
\end{array}\right) \in \mathbb{R}^{I \times I} .
$$

For the integral term we have the following ideas.

Case 1. Consider the following:

$$
\int_{0}^{t} \lambda_{2} c(x, t) d t \approx \lambda_{2} t c(x, t)
$$

and we obtain the matrix

$$
\Lambda_{2}=\left(\begin{array}{ccccc}
\frac{\lambda_{2} t^{2}}{2} & 0 & & & \\
0 & \frac{\lambda_{2} t^{2}}{2} & 0 & & \\
& \ddots & \ddots & \ddots & \\
& & 0 & \frac{\lambda_{2} t^{2}}{2} & 0 \\
& & & 0 & \frac{\lambda_{2} t^{2}}{2}
\end{array}\right) \in \mathbb{R}^{I \times I} .
$$

For the operator splitting scheme, we apply $A$ and $B=$ $-\Lambda_{1}+\Lambda_{2}$ and we apply the iterative splitting method, given in (21).

Case 2. We integrate the operator $B$ with respect to the previous solutions $\mathbf{C}_{i-1}, \Lambda_{2}\left(\mathbf{C}_{i-1}\right)$ and we obtain the matrix

For the reference solution, we apply a fine time and spatial scale without decoupling the equations. Figure 1 presents the numerical errors between the exact and the numerical solution. Here we obtain the optimal results for one-sided iterative schemes on the operator $B$, meaning that we iterate with respect to $B$ and use $A$ as the right-hand side.

Remark 7. For all iterative schemes, we can reach results faster than with the standard schemes, due to the fact that the iterative schemes benefit from their fast computations of the exponential matrices. With from four to five iterative steps, we obtain more accurate results than we did with 


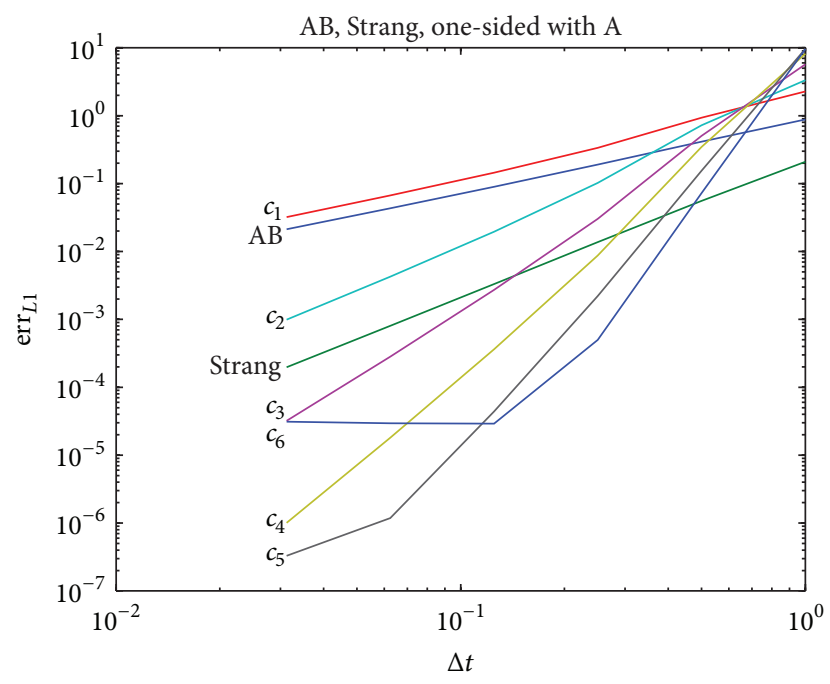

(a)

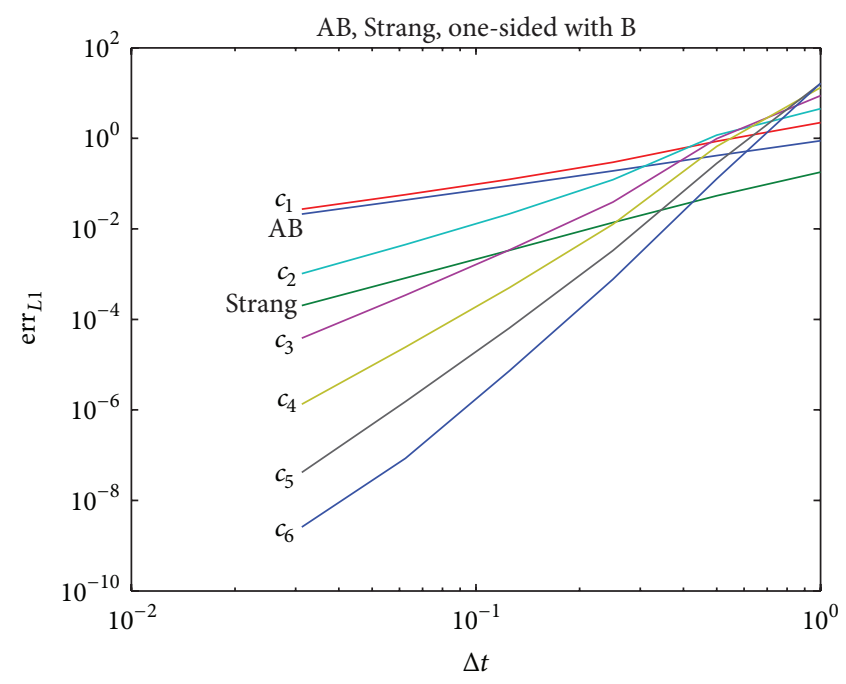

(b)

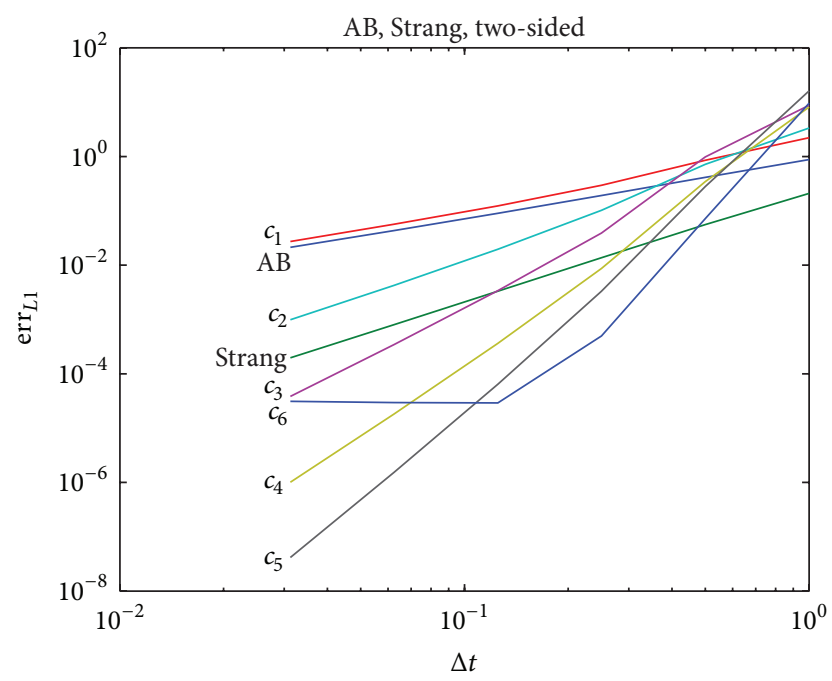

(c)

Figure 1: Numerical errors of the one-sided splitting scheme with $A$ (a), the one-sided splitting scheme with $B$ (b), and the iterative schemes with $1, \ldots, 6$ iterative steps (c).

the expensive standard schemes. With one-sided iterative schemes, we obtain the best convergence results.

\section{Conclusions and Discussion}

We presented the coupled model for the transport of deposition species in a plasma environment. We assumed that the flow field could be computed by the plasma model and the transport of the deposition species by a transport reaction model.

Such a first model can help understand the important modeling of the plasma environment in a CVD reactor.

\section{Conflict of Interests}

The author declares that there is no conflict of interests regarding the publication of this paper.

\section{References}

[1] N. S. J. Braithwaite and R. N. Franklin, "Reflections on electrical probes," Plasma Sources Science and Technology, vol. 18, no. 1, Article ID 014008, 2009.

[2] M. Lapke, T. Mussenbrock, and R. P. Brinkmann, "Modelling of volume- and surface wave based plasma resonance spectroscopy," in Proceedings of the Abstracts IEEE International Conference on Plasma Sciences, pp. 8-9, 2010.

[3] J. Oberrath, M. Lapke, T. Mussenbrock, and R. P. Brinkmann, "A functional analytical description of active plasma resonance spectroscopy in terms of kinetic theory," in Proceedings of the 30th International Conference on Phenomena in Ionized Gases (ICPIG '11), Belfast, Ireland, August 2011.

[4] S. Ukai and T. Yang, Mathematical Theory of Boltzmann Equation, Lecture Notes Series No 8, Liu Bie Ju Center of Mathematical Sciences, City University of Hong Kong, 2006. 
[5] K.-J. Engel and R. Nagel, One-Parameter Semigroups for Linear Evolution Equations, Springer, New York, NY, USA, 2000.

[6] A. Rhandi, Spectral Theory for Positive Semigroups and Applications, Quaderno Q. 1-2002, University of Lecce, Lecce, Italy, 2002.

[7] G. Strang, "On the construction and comparison of difference schemes," SIAM Journal on Numerical Analysis, vol. 5, pp. 506517, 1968.

[8] J. G. Verwer and B. Sportisse, "A note on operator splitting in a stiff linear case," Tech. Rep. MAS-R9830, CWI, 1998.

[9] Z. Zlatev, Computer Treatment of Large Air Pollution Models, Kluwer, 1995.

[10] J. Geiser, Splitting Methods for Differential Equations, Chapman \& Hall/CRC, 2011.

[11] E. Hansen and A. Ostermann, "Exponential splitting for unbounded operators," Mathematics of Computation, vol. 78, no. 267, pp. 1485-1496, 2009.

[12] S. Descombes and M. Thalhammer, "The LieTrotter splitting method for nonlinear evolutionary problems involving critical parameters. A compact local error representation and application to nonlinear Schrdinger equations in the semi-classical regime," IMA Journal of Numerical Analysis, vol. 33, no. 2, pp. 722-745, 2012.

[13] E. Zeidler, Nonlinear Functional Analysis and Its Applications. II/B Nonlinear Montone Operators, Springer, Berlin, Germany, 1990.

[14] J. F. Kanney, C. T. Miller, and C. T. Kelley, "Convergence of iterative split-operator approaches for approximating nonlinear reactive problems," Advances in Water Resources, vol. 26, no. 3, pp. 247-261, 2003. 


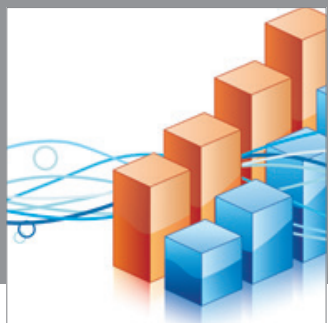

Advances in

Operations Research

mansans

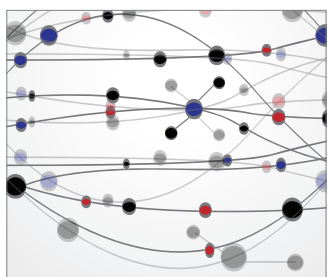

The Scientific World Journal
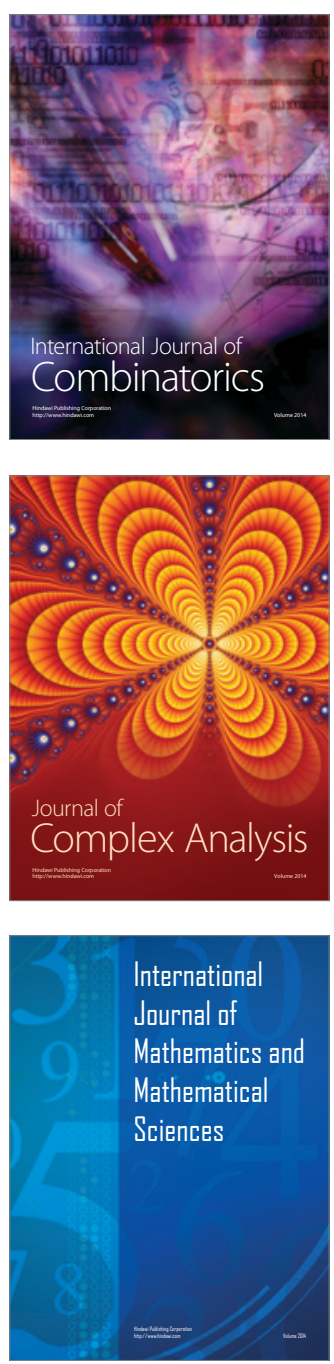
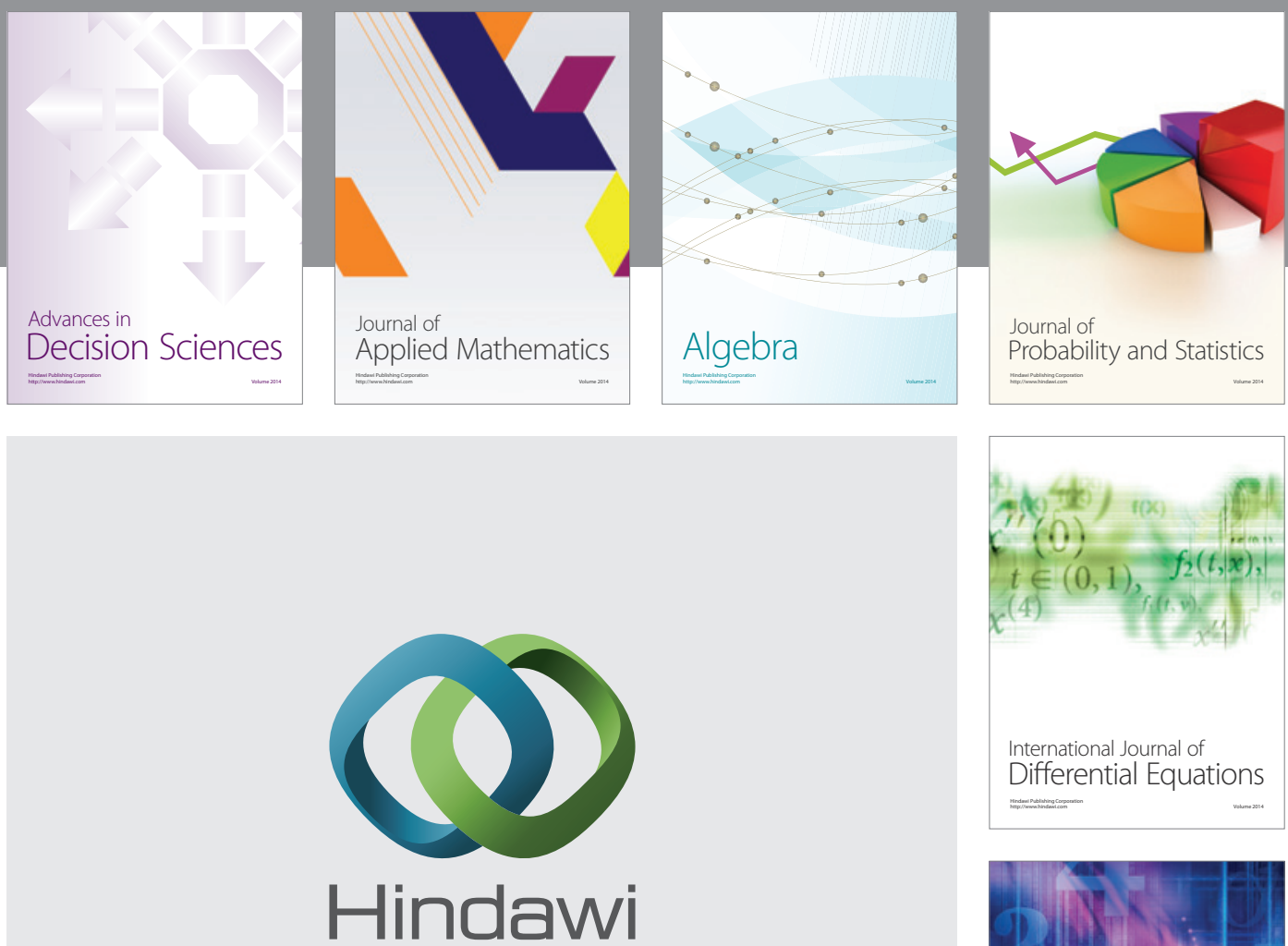

Submit your manuscripts at http://www.hindawi.com
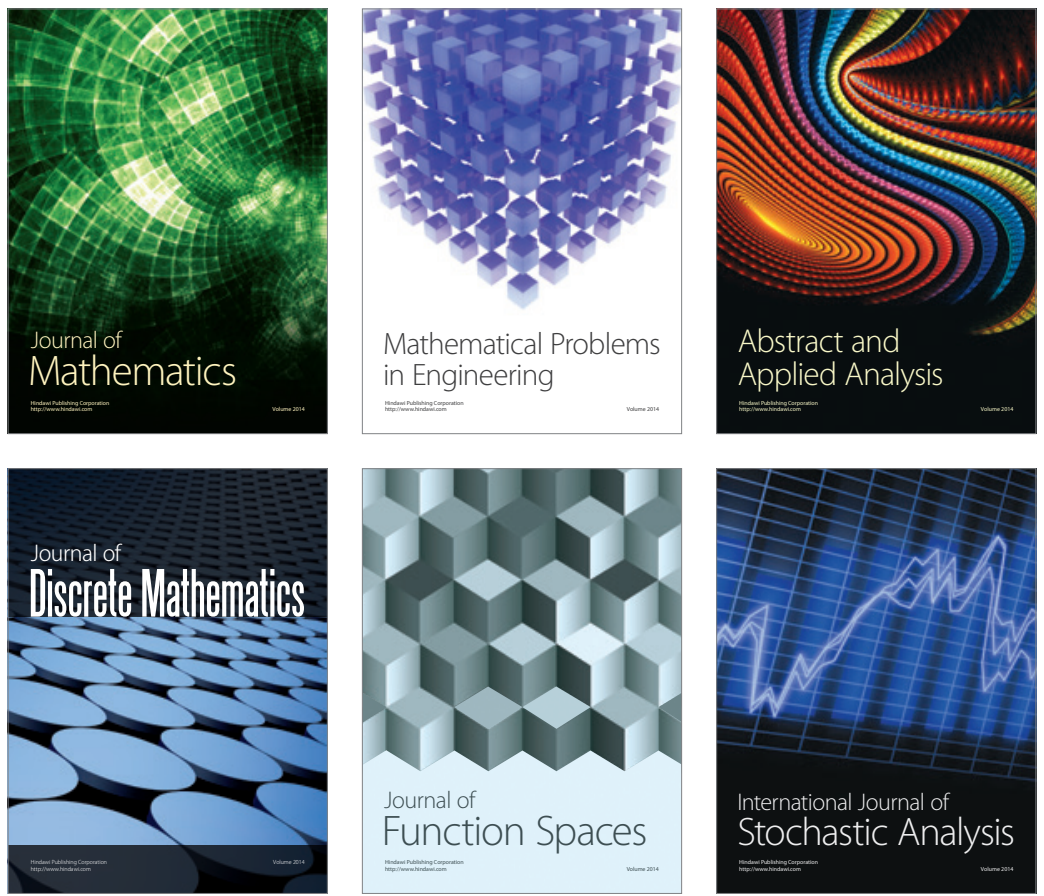

Journal of

Function Spaces

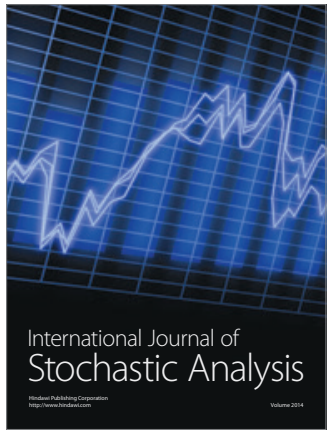

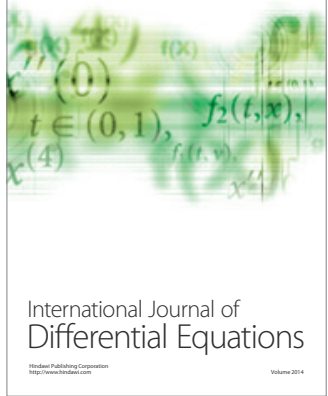
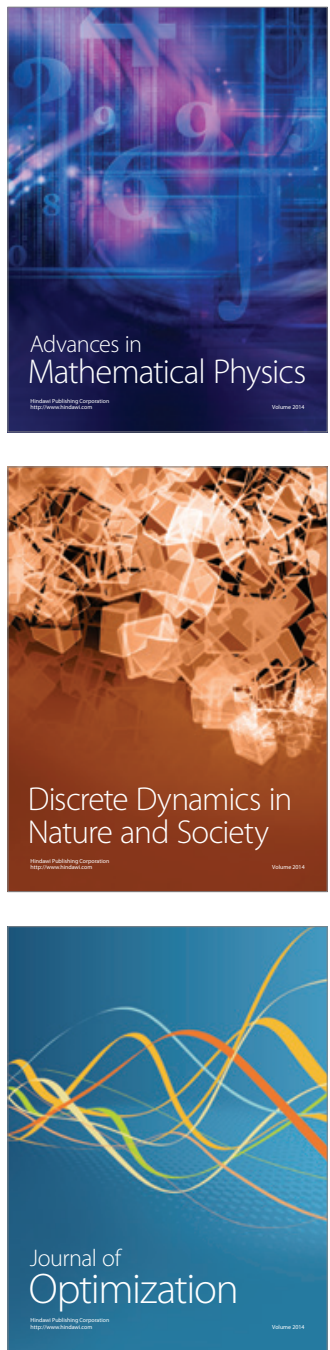